\title{
Percepções de aprendizado entre os alunos da Liga de Ginecologia
}

\author{
Perceptions of learning among the students of the League of Gynecology
}

\section{Leila Tiemi Okajima ${ }^{1}$, Laura Mattucci Tardelli ${ }^{1}$, Marina Miyuki Yamamoto Mota ${ }^{1}$, Maricy Tacla Alves Barbosa², Isabel Cristina Esposito Sorpreso², Edmund Chada Baracat ${ }^{3}$}

Okajima LT, Tardelli LM, Mota MMY, Barbosa MTA, Sorpreso ICE, Baracat EC. Percepções de aprendizado entre os alunos da Liga de Ginecologia / Perceptions of learning among the students of the League of Gynecology. Rev Med (São Paulo). 2019 jan.-fev.;98(1):72-6.

\begin{abstract}
RESUMO: Introdução: A Liga de Ginecologia atualmente conta com 8 membros, 4 diretores, 2 médicos assistentes e 1 residente, para o desenvolvimento de educação, pesquisa e extensão, para a disseminação de aprendizado na educação médica. Objetivo: Identificar as percepções de aprendizado dos acadêmicos da Liga de Ginecologia. Método: Trata-se de estudo transversal, realizado em agosto de 2017, na Faculdade de Medicina da Universidade de São Paulo. Foi utilizado um questionário respondido por membros da Liga de Ginecologia entre os anos de 2015 e 2017. Resultados: O principal motivo de ingresso na Liga é aprender o exame físico ginecológico, o principal aprendizado é a semiologia ginecológica, o principal ponto positivo é o atendimento ambulatorial supervisionado e o principal ponto negativo é a ausência de aulas teóricas. Além disso, $70 \%$ referiu influência da Liga na possibilidade de realizar residência médica em Ginecologia. Conclusão: As percepções de aprendizado entre os participantes da Liga de Ginecologia revelaram aquisições de competências em semiologia ginecológica e atendimento ambulatorial, lacuna de aulas teóricas na Liga e incentivo à pósgraduação.
\end{abstract}

Descritores: Educação médica; Ginecologia/educação; Saúde da mulher; Ensino; Educação de graduação em medicina; Estudantes de medicina.

\begin{abstract}
Background: The League of Gynecology currently has 8 members, 4 directors, 2 assistant physicians and 1 resident, for the development of education, research and extension, for the dissemination of learning in medical education. Objective: To identify the perceptions of learning among the academics of the League of Gynecology. Method: This is a cross-sectional study, carried out in August 2017 at the Faculty of Medicine of the University of São Paulo. A questionnaire answered by members of the League of Gynecology between the years of 2015 and 2017 was used. Results: The main reason to join the League is to learn the gynecological physical examination, the main learning is the gynecological semiology, the main positive point is supervised outpatient care and the main negative point is the absence of theoretical classes. In addition, $70 \%$ mentioned the influence of the League in the possibility of performing medical residency in Gynecology. Conclusion: Learning perceptions among the participants of the Gynecology League revealed the acquisition of skills in gynecological semiology and outpatient care, a gap in theoretical classes in the League and encouragement to postgraduation.
\end{abstract}

Keywords: Education, medical; Gynecology/education; Women's health; Teaching; Education, medical, undergraduate; Students, medical.

Trabalho realizado no Hospital das Clínicas da Faculdade de Medicina da Universidade de São Paulo (HCFMUSP), São Paulo, SP.

1. Acadêmicas de Medicina da Faculdade de Medicina da Universidade de São Paulo (FMUSP). Okajima LT - https://orcid.org/00000002-4482-6299; Tardelli LM - https://orcid.org/0000-0003-3731-1657; Mota MMY - https://orcid.org/0000-0002-0376-6621. Email: leila.okajima@fm.usp.br; laura.tardelli@fm.usp.br; marina.mota@fm.usp.br.

2. Professoras Doutoras da Disciplina de Ginecologia do Departamento de Obstetrícia e Ginecologia da Faculdade de Medicina da Universidade de São Paulo (FMUSP). Sorpreso ICE - https://orcid.org/0000-0002-5475-5957; Tacla M - https://orcid.org/0000-00022712-2724. Email: icesorpreso@usp.br; maricy.tacla@gmail.com.

3. Professor Titular da Disciplina de Ginecologia do Departamento de Obstetrícia e Ginecologia da Faculdade de Medicina da Universidade de São Paulo (FMUSP). https://orcid.org/0000-0003-0111-9030. Email: e.baracat@fm.usp.br.

Endereço para correspondência: Divisão de Clínica Ginecológica, Instituto Central do Hospital das Clínicas da FMUSP, $10^{\circ}$ andar. Av. Dr. Enéas Carvalho de Aguiar, 255 - Cerqueira César - São Paulo, SP - CEP 05403-000. Email: ligaapm@gmail.com. 


\section{INTRODUÇÃO}

$\mathrm{O}$ cuidado à mulher, antes concentrado no ciclo gravídico-puerperal, sofre transformações contínuas, principalmente com a entrada da mulher no mercado de trabalho, a redução da natalidade e o envelhecimento da população. Diante disso, os reflexos sobre o ensino médico são evidentes, em busca de estratégias de ensino e aprendizado sintonizadas com as condições atuais e as tendências futuras ${ }^{1}$.

Como instrumento a fim de acompanhar as mudanças no cenário da saúde, surgem as Ligas Acadêmicas, grupos voluntários de estudantes de Medicina de diferentes períodos da graduação, que, sob supervisão de especialistas, visam aprofundar o conhecimento e a prática sobre determinado assunto, com foco no trinômio: educação, pesquisa e extensão. As Ligas Acadêmicas estão entre as maiores responsáveis pela disseminação do aprendizado de diferentes temas nas universidades brasileiras ${ }^{2}$.

Na Faculdade de Medicina da Universidade de São Paulo (FMUSP), a primeira Liga Acadêmica registrada foi a de Combate a Sífilis e a Outras DST's, com mais de 90 anos de história. A formação de Ligas Acadêmicas se perpetua até os dias atuais, como iniciativa de acadêmicos para aperfeiçoar a própria educação médica ${ }^{3}$.

A Liga de Ginecologia da FMUSP compõe o conjunto de mais de 70 Ligas da instituição. O presente estudo tem como objetivo identificar as percepções de aprendizado entre alunos da Liga de Ginecologia.

\section{RELATO DE EXPERIÊNCIA DA LIGA DE GINECOLOGIA}

A Liga de Assistência Primária à Mulher da FMUSP foi fundada há 29 anos, em 1988. Há um ano, seu nome foi alterado para Liga de Ginecologia, visto que não realiza apenas atendimentos na assistência primária, mas também em outros níveis de atenção à saúde.

A Liga de Ginecologia tem como principal objetivo inserir gradualmente os alunos no atendimento ginecológico.

Os objetivos de aprendizado da Liga incluem: o desenvolvimento de competências para estabelecimento de uma relação médico-paciente humanizada, o aperfeiçoamento de habilidades de comunicação e empatia, as peculiaridades da anamnese em Ginecologia assim como as particularidades do exame físico ginecológico.

Para ingressar na Liga de Ginecologia, é necessário realizar uma prova, baseada em curso introdutório à Liga, que ocorre em três dias no $2^{\circ}$ semestre do ano. O curso não é obrigatório, no entanto, oferece ao aluno uma visão geral e didática sobre o atendimento e as principais queixas em Ginecologia.

A cada ano, são aceitos, em média, 8 novos membros, alunos do $2^{\circ}, 3^{\circ}, 4^{\circ}$ e $5^{\circ}$ ano de Medicina.

A organização é administrada pela diretoria da Liga, atualmente com quatro membros, um presidente e três diretores, que organizam a agenda, as atividades semanais, o curso introdutório, a frequência e a participação dos membros. Além disso, a Liga conta com dois médicos assistentes fixos e um médico residente que muda a cada três semanas.

As atividades da Liga acontecem às terças e sextas-feiras, das $12 \mathrm{~h}$ às $14 \mathrm{~h}$. Ocorrem, em média, dois atendimentos por dia. Os membros geralmente atendem em duplas, realizam a anamnese, discutem o caso com o médico assistente e/ou com o médico residente responsável e realizam o exame físico acompanhados pelo médico supervisor, em que apenas um aluno realiza o exame, sempre visando o respeito e o bem-estar da paciente. Em seguida, a melhor conduta é estabelecida. Em casos mais simples, a paciente retorna em consulta com a própria Liga, em casos mais complexos, a paciente é encaminhada para o setor da Ginecologia mais capacitado em acompanhar seu caso. De modo geral, a Liga atua como um ambulatório de primeira consulta e triagem.

As pacientes são funcionárias do Hospital das Clínicas da FMUSP ou pacientes encaminhadas de serviços de menor complexidade, de todas as faixas etárias. As pacientes são informadas sobre o funcionamento da Liga e aceitam ser atendidas pelos membros.

As principais queixas incluem rotina ginecológica, como colpocitologia oncótica (Papanicolaou) e mamografia, sintomas do climatério, sangramento uterino anormal, como miomas, dor pélvica, como dismenorreia e endometriose, e corrimentos vaginais, como vaginose bacteriana.

\section{MÉTODO}

Trata-se de estudo transversal, qualitativo e descritivo, realizado no período de agosto de 2017, na Liga de Ginecologia da Disciplina de Ginecologia da FMUSP.

\section{População}

A população foi composta por 34 graduandos do Curso de Medicina da FMUSP com atividade prévia ou atual na Liga de Ginecologia da Disciplina de Ginecologia da FMUSP entre os anos de 2015 e 2017.

Os critérios de inclusão foram: graduandos matriculados regularmente no Curso de Medicina com atividade prévia ou atual na Liga e participação voluntária.

Os critérios de não inclusão foram: ausência de contato ou resposta do membro da Liga.

\section{Procedimento de Coleta de Dados}

Foi enviado e-mail a todos os endereços cadastrados na Liga com enunciado de explicação sobre objetivos, riscos e benefícios do inquérito e anuência escrita em participar voluntariamente. 
Em anexo, o questionário (Anexo I), baseado em estudo semelhante realizado em alunos da Liga de Puericultura da FMSUP ${ }^{4}$, era composto por questões sobre os motivos em participar da Liga, os principais pontos positivos e negativos, os principais aprendizados, a possibilidade de realizar residência em Ginecologia e as sugestões de melhora do funcionamento da Liga. $\mathrm{O}$ participante respondia ao questionário em plataforma online. Não responderam ao questionário 24 indivíduos.

\section{Aspectos éticos}

Os aspectos éticos foram respeitados. Os participantes, após instrução, anuíram, por escrito, em participar voluntariamente do inquérito. Não houve divulgação de informações pessoais dos participantes do estudo ou constrangimento. $\mathrm{O}$ anonimato dos participantes foi assegurado pela pesquisadora e pelo instrumento de coleta de dados. O estudo teve anuência do responsável
Discente da Liga e do Conselho da Disciplina de Ginecologia do Departamento de Obstetrícia e Ginecologia da FMUSP.

\section{RESULTADOS}

O questionário foi respondido por 10 acadêmicos e apresentou como resultados os pontos discutidos a seguir.

Todos os alunos ressaltaram como interesse motivador para participar da Liga aprender o exame ginecológico. Além disso, 90\% citou entusiasmo em aprender sobre as principais queixas ginecológicas e conhecer a Ginecologia. Dentre os alunos, 80\% demonstrou interesse em aprender anamnese ginecológica. $70 \%$ em realizar atendimento ambulatorial, enquanto $40 \%$ e $30 \%$, respectivamente, responderam entrar em contato com pacientes precocemente e aperfeiçoar a comunicação e o vínculo médico-paciente, conforme mostra o Gráfico 1.

Gráfico 1. Principais motivos em participar da Liga de Ginecologia

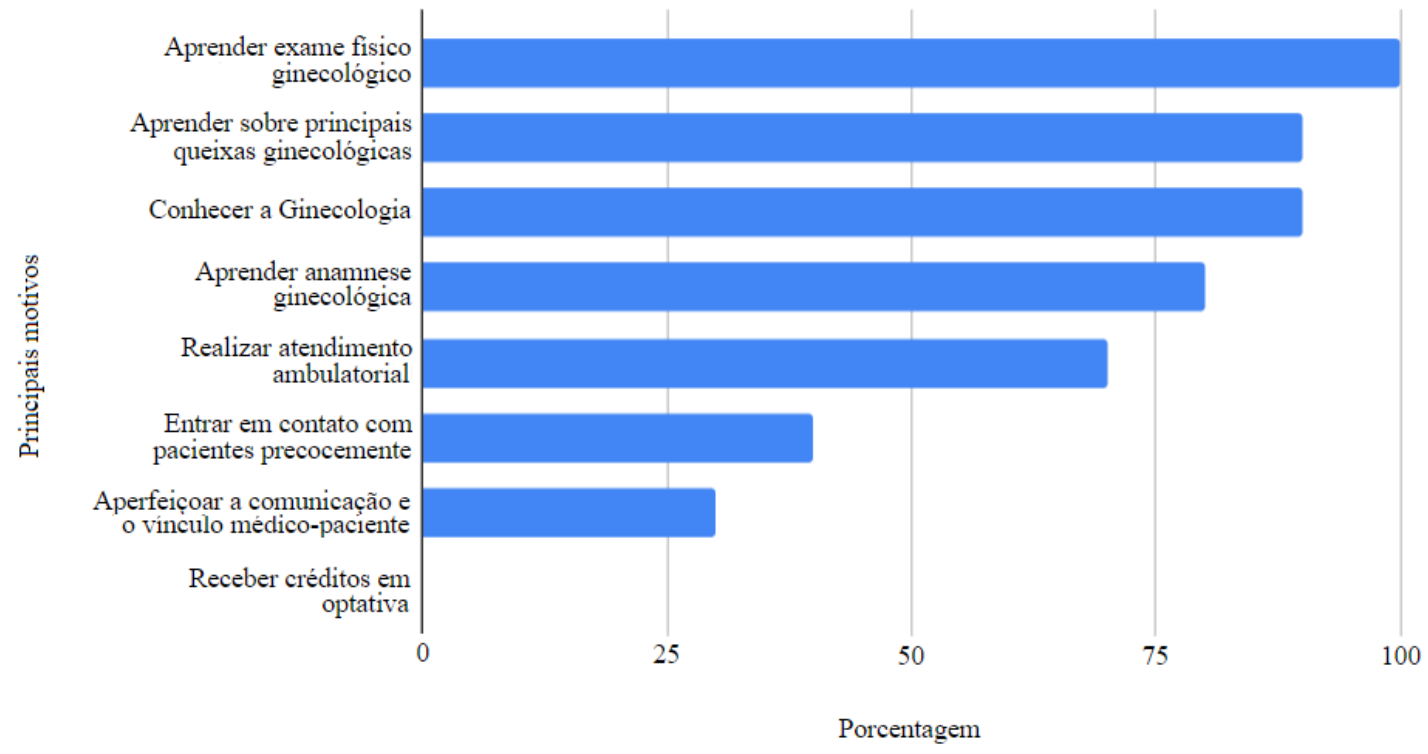

Os principais aprendizados da Liga, de acordo com os alunos, foram: propedêutica ginecológica, incluindo anamnese e exame físico, e as patologias mais comuns da Ginecologia.

Os pontos positivos ressaltados pelos alunos quanto a Liga foram: atendimento ambulatorial supervisionado, treino de exame ginecológico e coleta de exames complementares, boas discussões de casos.

Os pontos negativos ressaltados pelos alunos quanto a Liga foram: inexistência de aulas teóricas, poucas oportunidades de atendimento longitudinal, ajuda de apenas um residente por dia.

Quanto ao interesse na residência médica de Ginecologia e Obstetrícia, 40\% dos alunos demonstrou dúvida, $40 \%$ respondeu negativamente e $20 \%$ respondeu positivamente. $70 \%$ dos alunos referiu influência da Liga quanto a esse pensamento, dentro dos quais $80 \%$ positivamente.

Como sugestões para a Liga, maior número de pacientes, maior número de discutidores, acompanhamento de ultrassom, conização e histeroscopia, acompanhamento de cirurgias e aulas teóricas foram os principais pontos destacados pelos acadêmicos.

\section{DISCUSSÃO}

Alunos de Medicina buscam Ligas Acadêmicas motivados por ausência e/ou carência de natureza 
Okajima LT, et al. Percepções de aprendizado entre os alunos da Liga de Ginecologia.

curricular, por protagonismo de estudantes sensibilizados com as demandas da comunidade e por desejo de formação mais sólida ${ }^{5}$. Tais evidências vão ao encontro dos resultados do estudo, já que aspectos como aprender exame físico ginecológico, anamnese ginecológica e principais queixas em Ginecologia foram apontados como motivadores em participar da Liga e como principais aprendizados para uma formação mais sólida.

Destaca-se o aprendizado do exame físico ginecológico como principal motivador para a entrada de acadêmicos na Liga de Ginecologia. O exame físico ginecológico é pouco praticado durante a graduação, antes do internato, em respeito à intimidade das pacientes. Pacientes de hospital escola, de um modo geral, referem satisfação em contribuir para o ensino médico e afirmam receber tratamento educado, atencioso e respeitoso, no entanto, as áreas de Ginecologia e Urologia se mostram as mais delicadas quanto à presença de estudantes durante os atendimentos 6 . Por isso, a prática do exame físico ginecológico contribui tanto para o aprendizado dos alunos, como apontado nos resultados do estudo, quanto para o bem-estar da paciente, uma vez que possa ser realizado com técnica e rapidez. Ademais, o treinamento em comunicação médico-paciente é componente central na boa prática em Medicina ${ }^{7}$ e deve ser praticado durante o exame físico, para garantir comodidade à paciente.

Todavia, Ligas Acadêmicas oferecem tanto benefícios quanto riscos à formação médica ${ }^{8}$. É fundamental avaliar o funcionamento das Ligas para detectar possíveis desvios, como subversão da estrutura curricular, especialização precoce e reforço de vícios acadêmicos, o que pode refletir na formação médica. Devese sempre analisar a atuação das Ligas e manter a missão de desenvolver integralmente ensino, pesquisa e extensão para o bem da comunidade ${ }^{9}$.

Além disso, apesar do problema levantado de eventualmente haver poucos discutidores para os casos, o estudo mostra que as discussões de casos também foram proveitosas para o aprendizado dos alunos, levando a um treinamento para a passagem de história antes do internato e um maior conhecimento teórico sobre a Ginecologia. Este conhecimento teórico poderá ser aprofundado por aulas teóricas, conforme foi levantado como demanda de melhoria para a Liga.

Sendo assim, Ligas Acadêmicas são capazes de desenvolver habilidades e competências raramente estimuladas no currículo tradicional ${ }^{10-13}$, incluindo papel social, papel na prática médica, liderança, gestão, empreendedorismo, inovação, educação em saúde e construção da cidadania ${ }^{14}$, características trabalhadas tanto durante os atendimentos da Liga quanto durante a gestão da diretoria da Liga, que durante um ano cuida de sua organização. Portanto, o desenvolvimento e o incentivo de Ligas Acadêmicas nos currículos inovadores permitem treinamento necessário para que alunos de diferentes graduações em saúde, incluindo graduação em Medicina, adquiram habilidades necessárias para fornecer cuidados de alta qualidade em diferentes modelos de assistência médica $^{15}$.

Estas experiências curriculares de diferentes escolas médicas são encontradas em bases de dados, como PuBMed, EMBASE e SciELO, e em revisão sistemática com as palavras-chave "undergraduation study groups" e "teamworks and leaderships in medical school", sem uma correspondência detalhada de "Liga Acadêmica"16,17.

Como limitação do estudo, observa-se a reduzida amostra de participantes, uma vez que, em anos anteriores, não houve registro qualitativo e nem quantitativo sobre as percepções de aprendizado entre os membros da Liga.

Em contrapartida, a novidade é o retrato e o relato de experiência da Liga de Ginecologia, que se mantém há anos com formato assistencial direcionado à prática clínica $\mathrm{e}$ ao aprendizado no atendimento à saúde da mulher, englobando a mulher em período reprodutivo e não reprodutivo.

\section{CONCLUSÃO}

As percepções de aprendizado entre os membros da Liga de Ginecologia revelaram aquisições de competências em propedêutica ginecológica e atendimento ambulatorial, lacuna de aulas teóricas na Liga e incentivo à pósgraduação.

Conclui-se, portanto, em consonância com estudos prévios, que as Ligas Acadêmicas têm um papel relevante e complementar ao ensino da grade curricular universitária.

\section{Anexo I. Questionário - Liga de Ginecologia}

1. O que o motivou a participar da Liga de Ginecologia?

( ) Conhecer a Ginecologia

( ) Realizar atendimento ambulatorial

( ) Entrar em contato com pacientes precocemente

( ) Aperfeiçoar a comunicação e o estabelecimento de vínculo médico-paciente

( ) Aprender anamnese ginecológica

( ) Aprender exame físico ginecológico

( ) Aprender sobre as principais queixas ginecológicas

( ) Receber créditos em optativa

( ) Outro:

2. Pontos positivos da Liga de Ginecologia:

3. Pontos negativos da Liga de Ginecologia:

4. Principais aprendizados na Liga de Ginecologia:

5. Você pensa em fazer residência médica em Ginecologia?

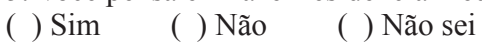

A Liga de Ginecologia o influenciou nesse pensamento?

( ) Sim ( ) Não

Se influenciou, de que forma?
( ) Positiva
( ) Negativa
6. Sugestões para a Liga de Ginecologia: 
Participação dos autores: Okajima LT, Tardelli LM, Mota MMY - levantamento da literatura, coleta de dados e confecção do manuscrito. Sorpreso ICE, Barbosa MTA - levantamento da literatura, coleta de dados, confecção do manuscrito e revisão. Baracat EC - levantamento da literatura, coleta de dados, confecção do manuscrito e revisão final.

\section{REFERÊNCIAS}

1. Amaral E, Azevedo GD, Abbade J. The teaching and learning of gynecology and obstetrics at the undergraduate level: challenges and trends. Rev Bras Ginecol Obstet. 2007;29(11):551-4. doi: http//dx.doi.org/10.1590/S010072032007001100001 .

2. Simões RL, Bermudes FAM, Andrade HS, Barcelos FM, Rossoni BP, Miguel GPS, Fagundes CAC, Fraga GP. Trauma leagues: an alternative way to teach trauma surgery to medical students. Rev Col Bras Cir. 2014;41(4):297-302. doi: http:// dx.doi.org/10.1590/0100-699120140040013.

3. Cavalieri VA, Figueiredo EG, Teixeira MJ. Neurosurgical League, an experience of twenty years. Rev Med (São Paulo). 2016;95(1):30-2. doi: http://dx.doi.org/10.11606/issn.16799836.v.95ilp30-32.

4. Shinzato MS, Carvalho MPDC, Gonzalez DC, Kuhne ACA, Ferrer APS, Sucupira ACSL. League childcare. Rev Med (São Paulo). 2013;92(1):57-61. doi: http://dx.doi.org/10.11606/ issn.1679-9836.v92i1p57-61.

5. Silva SA, Flores O. Academic leagues in student training. Rev Bras Educ Med. 2015;39(3):410-25. doi: http://dx.doi. org/10.1590/1981-52712015v39n3e02592013.

6. Berwanger J, Geroni GD, Bonamigo EL. Medical students in the perception of patients. Rev Bioét (Brasília). 2015;23(3):552-62. doi: http://dx.doi.org/10.1590/198380422015233092 .

7. Sanson-Fisher R, Hobden B, Waller A, Dodd N, Boyd L. Methodological quality of teaching communication skills to undergraduate medical students: a mapping review. BMC Med Educ. 2018;18(1):151. doi: http://dx.doi.org/10.1186/ s12909-018-1265-4.

8. Filho PTH, Villas-Bôas PJF, Corrêa FG, Muñoz GOC, Zaba M, Venditti VC, Schellini SA. Regulation of student leagues: the experience at the Botucatu School of Medicine. Rev Bras Educ Med. 2010;34(1):160-7. doi: http://dx.doi.org/10.1590/ S0100-55022010000100019.

9. Filho, PTH. Student Leagues: motivations and criticisms regarding a necessary reconsideration. Rev Bras Educ Med.
2011;35(4):535-43. doi: http://dx.doi.org/10.1590/S010055022011000400013 .

10. The Accreditation Council on Graduate Medical Education Outcomes Project [cited 2018 Aug 01]. Available from: www. acgme.org.

11. Institute of Medicine (US) Committee of Quality Health Care in America. Crossing the quality chasm: a new health system for the 21st century. Washington (DC): National Academy Press (US); 2001.

12. Sanson-Fisher R, Maguire P. Should skills in communicating with patients be taught in medical schools? Lancet. 1980;316(8193):523-6.

13. Pauli HG. Education and training of the physician: undergraduate medical education and health care. J Med Educ. 1973;48(12):51-5.

14. Ferreira DAV, Aranha RN, de Souza MHFO. Academic leagues: a brazilian way to teach about cancer in medical universities. BMC Med Educ. 2015;15:236. doi: http://dx.doi. org/10.1186/s12909-015-0524-X.

15. Neves FBCS, Vieira PS, Cravo EA, Dias M, Bitencourt A, Guimarães HP, Filho GSF, Orlando JMC. Survey on brazilian critical care medicine undergraduate study groups. Rev Bras Ter Intens. 2008;20(1):43-48. doi: http://dx.doi.org/10.1590/ S0103-507X2008000100007.

16. O'Connell MT, Pascoe JM. Undergraduate medical education for the 21st century: leadership and teamwork. Fam Med. 2004;36:S51-6.

17. Chakraborti C, Boonyasai RT, Wright SM, Kern DE. A systematic review of teamwork training interventions in medical student and resident education. J Gen Intern Med. 2008;23(6):846-53. doi: http://dx.doi.org/10.1007/s11606008-0600-6.

Recebido: 11.10 .17

Aceito: 28.03 .19 